\title{
Adjuvant treatment of breast cancer in postmenopausal women: role of exemestane
}

This article was published in the following Dove Press journal:

Breast Cancer:Targets and Therapy

13 October 2010

Number of times this article has been viewed

\author{
lain RJ Macpherson ${ }^{1,3}$ \\ Colin Lindsay ${ }^{2}$ \\ Peter Canney ${ }^{3}$ \\ 'Centre for Oncology and Applied \\ Pharmacology, University of Glasgow, \\ Glasgow, Scotland, UK; ${ }^{2}$ Beatson \\ Institute for Cancer Research, \\ Glasgow, Scotland, UK; ${ }^{3}$ Beatson West \\ of Scotland Cancer Centre, Glasgow, \\ Scotland, UK
}

\begin{abstract}
Adjuvant hormonal therapy significantly reduces the risk of recurrence and death following surgery for estrogen receptor (ER)-positive early breast cancer. Previously, the gold standard hormonal therapy was tamoxifen, a selective modulator of the ER. However, large clinical trials conducted over the past decade have defined the efficacy of an alternative class of hormonal agent, namely the third-generation aromatase inhibitors (AIs): exemestane, letrozole, and anastrozole. On the basis of the pivotal International Exemestane Study (IES), exemestane, a steroidal third-generation AI, was licensed for the adjuvant treatment of postmenopausal women with ER-positive early breast cancer following 2-3 years of tamoxifen to complete a total of 5 years of adjuvant hormonal therapy. Here, we consider recent data to emerge primarily from the IES and Tamoxifen Exemestane Adjuvant Multicenter trials and consider their implications for the role of exemestane in the adjuvant treatment of breast cancer.
\end{abstract}

Keywords: breast cancer, exemestane, aromatase, tamoxifen

\section{Introduction}

Breast cancer remains a major global health problem with 1.1 million new cases and 400,000 deaths occurring each year. ${ }^{1}$ The majority of breast cancers express the estrogen receptor (ER), are dependent on estrogen for growth and survival, and thus are potentially sensitive to manipulations that interfere with this pathway. Indeed, estrogen deprivation as a treatment for advanced breast cancer dates back to 1896 with George Beatson's demonstration of tumor regression in premenopausal women treated with bilateral oophorectomy. ${ }^{2}$ However, a true mechanistic understanding of this phenomenon would not be possible until the discovery of estrogen in the following century. Attempts to medically block estrogen biosynthesis culminated in the development of aminoglutethamide, a first-generation aromatase inhibitor (AI), in the $1970 \mathrm{~s}^{3}$ At the same time, tamoxifen, a selective modulator of the ER, was under development. ${ }^{4}$ In subsequent head-to-head trials in patients with advanced breast cancer, tamoxifen demonstrated similar efficacy but improved tolerability in comparison to aminoglutethamide. ${ }^{3}$ The ensuing development of tamoxifen in the adjuvant setting has been one of the key developments in the treatment of breast cancer. Indeed, it is now apparent from the Early Breast Cancer Trialists' Collaborative Group (EBCTCG) metaanalysis that around 5 years of adjuvant tamoxifen is associated with a $41 \%$ reduction in the annual recurrence rate and a $34 \%$ reduction in breast cancer-specific mortality. ${ }^{5}$

Aromatase, a member of the cytochrome P-450 family, catalyzes the final step in estrogen biosynthesis: the conversion of the androgens, androstenedione, and
Correspondence: lain RJ Macpherson Centre for Oncology and Applied Pharmacology, University of Glasgow, Cancer Research UK Beatson Laboratories, Switchback Road, Glasgow G6I IBD, UK

Tel +44 I4I 30 I 7 I 25

Fax +44 I4I 30I 7124

Email i.macpherson@beatson.gla.ac.uk 
testosterone to the estrogens, estrone, and estradiol, respectively. ${ }^{6}$ In premenopausal women, the ovaries are the major site of estrogen biosynthesis and aromatase is expressed in the granulosa cells of the ovarian follicle. Ovarian aromatase is regulated within a powerful hypothalamic-pituitary feedback loop, and AIs are not able to robustly downregulate estrogen biosynthesis in the premenopausal woman. However, a distinct situation exists in postmenopausal women in whom ovarian production of estrogen ceases. Most circulating estrogen then derives from the conversion of circulating ovarian and adrenal androgens by aromatase expressed in adipose tissue and muscle. In addition, aromatase is expressed by both malignant and stromal cells in many breast tumors, resulting in local concentrations of estrogen far exceeding the circulating levels. ${ }^{3,6}$

Aminoglutethamide had a relatively low specificity with resulting off-target effects (blockade of 11 1 -hydroxylase necessitating cortisol replacement) and limited potency. However, the current third-generation AIs are highly potent and specific, with in vivo assays indicating 97.3\%-99.1\% inhibition of whole-body aromatase activity., ${ }^{7,8}$ The thirdgeneration AIs fall into two distinct classes:

\section{Steroidal inhibitors: Exemestane}

Exemestane is an analog of the steroid androstenedione. It interacts with the steroid-binding region of the aromatase enzyme and forms an unbreakable covalent bond resulting in irreversible inhibition.

2. Nonsteroidal inhibitors: Anastrozole/Letrozole

These drugs competitively inhibit the hydroxylation reaction catalyzed by aromatase by binding with the heme iron of cytochrome P-450.

Several studies have confirmed a greater efficacy for third-generation AIs in comparison to tamoxifen in the advanced disease setting, thus prompting their investigation at earlier stages of disease..$^{9-11}$

\section{Exemestane}

Phase I studies of exemestane in healthy volunteers and breast cancer patients have demonstrated dose-dependent inhibition of plasma estrogen levels up to the $25 \mathrm{mg} / \mathrm{d}$ dose level, with no further inhibition achieved with doses up to $800 \mathrm{mg} .{ }^{12,13}$ Orally administered exemestane is rapidly absorbed, taking 2 hours for maximal concentrations to be reached. ${ }^{14}$ Exemestane is metabolized by the cytochrome P450 3A4 (CYP3A4) isoenzyme and by aldoketoreductases, followed by conjugation and excretion in urine and feces. ${ }^{15}$ The terminal half-life is around 27 hours. Inhibition of CYP3A4 was not associated with increased exposure to exemestane although drugs that induce CYP3A4 may reduce exposure to exemestane and efficacy. ${ }^{15}$ Following a single dose of exemestane, maximal suppression of estrogen was observed at 3 days and persisted for at least 5 days, consistent with a mechanism of irreversible enzyme inhibition. ${ }^{12}$ With daily dosing, steady-state levels are achieved in 7 days. ${ }^{14}$

Investigation of exemestane in patients with advanced breast cancer demonstrated superiority to megestrol acetate and tamoxifen and confirmed activity against cancers resistant to tamoxifen or third-generation nonsteroidal AIs. ${ }^{16-20}$

\section{Adjuvant trials of exemestane}

Given the superior efficacy of exemestane and other thirdgeneration AIs in women with advanced breast cancer, it was a priority to delineate their efficacy in the adjuvant setting. In this regard, a variety of strategies by which AIs could either replace or be combined with tamoxifen have been investigated. These include up-front, sequential, switch, and extended strategies (Figure 1). In the case of exemestane, the most informative trials have been the International Exemestane Study (IES) and the Tamoxifen Exemestane Adjuvant Multicenter (TEAM) study.

\section{International exemestane study}

This study randomized 4,724 postmenopausal women who were disease free after 2-3 years of adjuvant tamoxifen to receive either exemestane $(25 \mathrm{mg} / \mathrm{d})$ or further tamoxifen $(20-30 \mathrm{mg} / \mathrm{d})$ to achieve a total duration of endocrine therapy of 5 years. $^{21}$ The allocation of treatment was double blind. Inclusion criteria permitted entry of patients with either ER-positive or ER-unknown tumors. Just over half of the women in both arms had node-negative disease, and one-third had received adjuvant chemotherapy. The primary endpoint was disease-free survival (DFS; time from randomization to recurrence of breast cancer at any site, diagnosis of a second primary breast cancer, or death from any cause). Secondary endpoints included overall survival (OS) and tolerability.

Outcome data from IES were first published in 2004 on the recommendation of the independent data monitoring and safety committee following the second planned interim analysis, which was triggered by the occurrence of half the total number of planned events. ${ }^{21}$ In this analysis, the unadjusted hazard ratio (HR) in the exemestane group as compared with the tamoxifen group was 0.68 (95\% confidence interval [CI]: $0.56-0.82 ; P<0.001)$, representing an absolute benefit in DFS of $4.7 \%$ (95\% CI: $2.6-6.8)$ at 3 years after randomization (Table 1). There was no difference in OS although the number of events was low. Because this 
0 years 5 years

1. Tamoxifen monotherapy

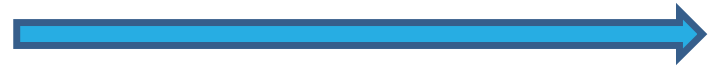

2. Al monotherapy

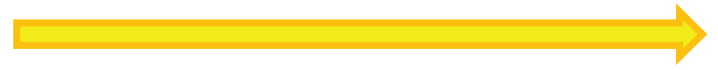

3. Sequence/Switch

(a) (b)

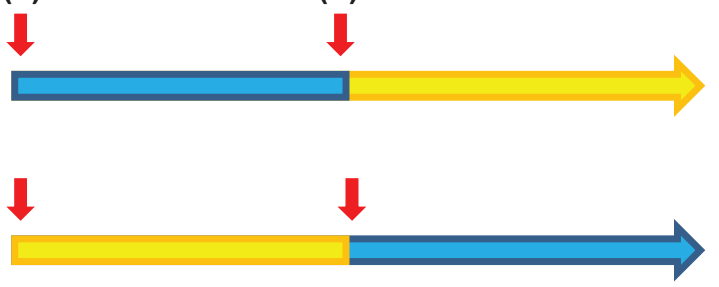

(c)

\section{Extended adjuvant}

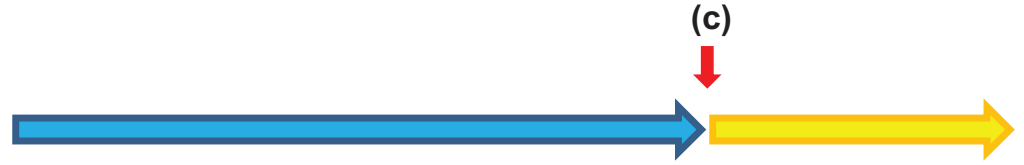

\section{Tamoxifen \\ Aromatase inhibitor}

Figure I Adjuvant Al clinical trial designs. Various strategies by which Als may be incorporated into adjuvant therapy have been investigated. Trials of Al monotherapy have typically randomized patients before commencing adjuvant therapy to either 5 years of Al or tamoxifen. The switching strategy entails a randomization to either tamoxifen or Al after 2.5-3 years of tamoxifen. Crucially, randomization at this time point (b) provides no information on events occurring before the switch. This differs from the sequencing trials in which randomization occurs at the initiation of adjuvant therapy (a). Extended adjuvant trials have randomized patients who have completed 5 years of adjuvant tamoxifen to an $\mathrm{Al}$ or not (c). As with the switch strategy, events occurring before randomization are not addressed.

treatment effect exceeded protocol-defined early stopping rules, early release of efficacy data was undertaken. By this point, more than $90 \%$ of patients had completed their planned treatment, thus diminishing the risk of confounding by nonprotocol-permitted crossover. At the time of this analysis, ER status was known for around $82 \%$ of cases, with just over $1 \%$ proving ER negative. The independent data monitoring and safety committee and the trial steering group agreed to perform a further analysis when $95 \%$ of patients had at least 3 years of follow-up. In addition, efforts were made to obtain ER status in as many of the ER-unknown patients as possible, and a supplementary analysis excluding those patients who enrolled with ER-unknown disease and were subsequently found to have ER-negative disease was prespecified.

This second analysis was published in $2007 .{ }^{22}$ Now with a median follow-up of 55.7 months, the unadjusted HR for DFS on an intention to treat basis was 0.76 (95\% CI: 0.66-0.88; $P=0.0001)$ in favor of exemestane (Table 1). This translated into a 3.3\% absolute improvement in DFS at 2.5 years after randomization. At 5-year postrandomization, this benefit was maintained with an absolute improvement in DFS of 3.4\%. Breast cancer-free survival, time to distant recurrence, and time to contralateral breast cancer were all significantly improved by switching to exemestane. The beneficial effect of exemestane on DFS appeared consistent across predefined subgroups according to age, nodal status, and previous chemotherapy. There had been 222 deaths in the exemestane group and 261 deaths in the tamoxifen group $(\mathrm{HR}=0.85$; 95\% CI: $0.71-1.02 ; P=0.08)$. An additional analysis excluding 122 patients subsequently found to be ER negative demonstrated a $17 \%$ reduction in the relative risk of death $(\mathrm{HR}=0.83 ; 95 \% \mathrm{CI}: 0.69-1.00 ; P=0.05)$.

An updated analysis, with median follow-up of 91 months, was presented at the European CanCer OrganisationEuropean Society for Medical Oncology congress in September $2009 .{ }^{23}$ In the intention-to-treat analysis, 
Table I Analyses of International exemestane study

\begin{tabular}{|c|c|c|c|}
\hline Publication/presentation & March 2004'1 & February $2007^{22}$ & September $2009^{23}$ \\
\hline Median follow-up & $30.6 \mathrm{mo}$ & $55.7 \mathrm{mo}$ & $91 \mathrm{mo}$ \\
\hline DFS events & 183 vs 266 & 354 vs 455 & 552 vs 641 \\
\hline DFS HR & $\begin{array}{l}0.68 \text { (95\% Cl: 0.56-0.82; } \\
P<0.00 I)\end{array}$ & $\begin{array}{l}0.76 \text { (95\% Cl: } 0.66-0.88 \\
P=0.000 \mathrm{I})\end{array}$ & $\begin{array}{l}0.84 \text { (95\% Cl: } 0.75-0.94 \\
P=0.002)\end{array}$ \\
\hline Absolute DFS benefit & $\begin{array}{l}4.7 \%(95 \% \mathrm{Cl}: 2.6-6.8) \text { at } 3 y \\
\text { postrandomization }\end{array}$ & $\begin{array}{l}3.4 \%(95 \% \mathrm{Cl}: 0.1-6.8) \text { at } 5 \mathrm{y} \\
\text { postrandomization }\end{array}$ & $\begin{array}{l}2.8 \%(95 \% \mathrm{Cl}: \mathrm{I} . \mathrm{I}-4.4) \\
\text { at } 5 \text { y postrandomization }\end{array}$ \\
\hline Breast cancer-free survival & $\begin{array}{l}\mathrm{HR}=0.63(95 \% \mathrm{Cl}: 0.5 \mathrm{I}-0.77 \\
P=0.0000 \mathrm{I})\end{array}$ & $\mathrm{HR}=0.76(95 \% \mathrm{Cl}: 0.65-0.89)$ & - \\
\hline Time to distant recurrence & - & $\mathrm{HR}=0.83$ (95\% Cl: $0.7 \mathrm{I}-0.99)$ & - \\
\hline Time to contralateral breast cancer & $\mathrm{HR}=0.44(95 \% \mathrm{Cl}: 0.20-0.98) 0.04$ & $\mathrm{HR}=0.57(95 \% \mathrm{Cl}: 0.33-0.98)$ & - \\
\hline OS (ITT) & $\begin{array}{l}\mathrm{HR}=0.88(95 \% \mathrm{Cl}: 0.67-1.16 \\
P=0.37)\end{array}$ & $\begin{array}{l}\mathrm{HR}=0.85(95 \% \mathrm{Cl}: 0.7 \mathrm{I}-\mathrm{I} .02 \\
P=0.08)\end{array}$ & $\begin{array}{l}\mathrm{HR}=0.89(95 \% \mathrm{Cl}: 0.77-\mathrm{I} .02 \\
P=0.09)\end{array}$ \\
\hline OS (ER positive/unknown) & - & $\begin{array}{l}\mathrm{HR}=0.83(95 \% \mathrm{Cl}: 0.69-1.00 \\
P=0.05)\end{array}$ & $\begin{array}{l}\mathrm{HR}=0.86 \text { (95\% Cl: 0.75-0.99; } \\
P=0.04)\end{array}$ \\
\hline
\end{tabular}

Abbreviations: DFS, disease-free survival; HR, hazard ratio; $\mathrm{Cl}$, confidence interval; OS, overall survival; ITT, intention-to-treat; ER, estrogen receptor; mo, months; $y$, years.

a significant improvement in DFS and trend to improvement in OS were seen to be maintained several years after cessation of treatment (Table 1). When patients with ER-negative disease were excluded, a significant improvement in OS was now apparent. This corresponded to an absolute difference in OS of $1.4 \%$ at 5 years and $2.4 \%$ at 8 years.

In summary, IES has demonstrated a reduction in all types of DFS events for patients switched to exemestane after 2-3 years of tamoxifen vs patients remaining on tamoxifen. Furthermore, with longer follow-up and with the prespecified exclusion of patients recruited with unknown ER status and subsequently found to be ER negative, an improvement in OS has been established.

\section{TEAM trial}

The TEAM trial was initiated in 2001 as an open-label randomized comparison of exemestane ( $25 \mathrm{mg} /$ daily) vs tamoxifen (20 mg/daily) as initial adjuvant therapy. However, following the publication of interim results of the IES trial in 2004, the protocol was modified such that all patients on the tamoxifen arm would switch to exemestane after 2.5-3 years. The modified study design included 2 co-primary endpoints: 1) DFS of tamoxifen vs exemestane at 2.75 years of median follow-up (the choice of 2.75 years represents the midpoint of the planned tamoxifen to exemestane crossover window; therefore, this analysis is essentially a test of upfront exemestane vs tamoxifen) and 2) DFS at 5 years of exemestane vs the tamoxifen-exemestane sequence. Events contributing to DFS were loco-regional or distant breast cancer recurrence, second primary or contralateral breast cancer, and death from any cause. Secondary endpoints included OS, relapse-free survival (RFS), and time to distant metastases.
Only postmenopausal women with known ER and/or progestogen receptor (PR)-positive disease were eligible.

Almost 10,000 women were randomized to receive either upfront exemestane $(n=4,904)$ or the tamoxifen-exemestane sequence $(n=4,875)$. One hundred percent of cases were ER positive and/or PR positive, and $47 \%$ were node positive. Approximately one-third of patients in each arm had received adjuvant chemotherapy.

Results for the first co-primary endpoint were presented at the San Antonio Breast Cancer Symposium in $2008 .{ }^{24}$ For this analysis, all patients were censored at 2.75 years. Three hundred fifty-two DFS events had occurred in the exemestane arm vs 388 events in the tamoxifen arm, giving a HR of 0.89 (95\% CI: $0.77-1.03 ; P=0.12$ ). Although the primary endpoint was not met, predefined secondary endpoints did achieve statistical significance with a HR of $0.85(95 \% \mathrm{CI}$ : $0.72-1.0 ; P=0.05$ ) for RFS and 0.81 (95\% CI: $0.67-0.98$; $P=0.03$ ) for time to distant metastases in favor of exemestane. A potential confounder of these data is the significant proportion of patients who failed to complete 2.75 years of treatment. In particular, 1,434 (29.5\%) patients randomized to the tamoxifen-exemestane sequence failed to complete 2.75 years of treatment, with 754 patients switching prematurely to exemestane.

The second co-primary endpoint (DFS at 5 years for upfront exemestane vs a tamoxifen-exemestane sequence) was presented at the San Antonio meeting in December 2009. ${ }^{25}$ The median follow-up was 5.1 years, with $60 \%$ having completed 5 years of follow-up. In this analysis, no benefit was demonstrated for upfront exemestane over the tamoxifenexemestane sequence. Specifically, no improvement in DFS [HR 0.97 (95\% CI: $0.88-1.08 ; P=0.604)$ ] or in OS 
(HR $=1.0$ [95\% CI: 0.89-1.14; $P=0.99]$ ) was observed. Although the data are relatively immature, particularly for OS, the lack of any signal would suggest that a significant difference between the arms is unlikely to emerge. In this context, it is pertinent to consider the results of Breast International Group (BIG) 1-98, which similarly evaluated an upfront AI (letrozole) vs sequenced therapy incorporating tamoxifen. In this comparison, DFS was not significantly improved by sequenced therapy vs letrozole alone. ${ }^{26} \mathrm{~A}$ nonstatistically significant trend toward worse outcomes was observed in those patients randomized to tamoxifen followed by letrozole in comparison to letrozole monotherapy (HR $=1.05$; 99\% CI: $0.84-1.32$ ) but not for the reverse sequence of letrozole followed by tamoxifen $(\mathrm{HR}=0.96 ; 99 \%$ CI: 0.76-1.21). Reminiscent of the TEAM data, there were more early relapses in women randomized to tamoxifen followed by letrozole rather than letrozole alone. ${ }^{26}$

\section{National Surgical Adjuvant Breast and Bowel Project B33 trial}

In this randomized double-blind placebo-controlled trial, patients who were disease free after completing 5 years of adjuvant tamoxifen were randomly assigned to receive 5 years of exemestane $(25 \mathrm{mg} / \mathrm{d})$ or matched placebo. ${ }^{27}$ The primary endpoint was DFS (events defined as local or distant recurrence, second primary cancer, and death from any cause). Secondary endpoints were RFS, OS, and toxicity. Almost 1,600 of a planned 3,000 patients were recruited between May 2001 and October 2003. However, recruitment was terminated following the publication of data from the MA17 trial, which demonstrated a $42 \%$ reduction in the risk of recurrence for letrozole vs placebo in a similar patient population. Patients on study were unblinded, and those in the placebo group were offered exemestane. Forty-four percent of eligible patients receiving placebo opted to switch to exemestane although for the majority of these patients, this switch took place 6-12 months after unblinding. Despite the early closure of this trial and significant crossover from placebo to exemestane, the intention to treat primary analysis demonstrated a trend toward improved DFS $(\mathrm{HR}=0.68$; $P=0.07)$ together with a statistically significant improvement in RFS (HR $=0.44 ; P=0.004)$ in those patients initially allocated exemestane.

\section{ATENA Trial}

The Adjuvant Post-Tamoxifen Exemestane (ATENA) trial was a randomized open-label study initiated by the Hellenic
Society of Breast Surgeons to compare observation in 5 years of exemestane vs observation in postmenopausal woman who had completed 5-7 years of adjuvant tamoxifen. ${ }^{27}$ However, the ATENA trial was terminated having randomized 448 of a planned 1,800 patients following publication of the results of the MA17 trial.

\section{MA27}

The ongoing Canadian NCIC-MA27 trial randomized postmenopausal women with early breast cancer to receive adjuvant either anastrozole $1 \mathrm{mg} /$ daily or exemestane $25 \mathrm{mg}$ /daily for 5 years. ${ }^{28}$ The primary endpoint is event-free survival, and substudies to compare the effects of these drugs on bone mineral density (BMD) and breast density have been incorporated. Recruitment is now completed.

\section{Toxicities of exemestane}

The adverse effects of exemestane in this patient population have been characterized in large Phase III trials and their respective substudies and appear broadly similar to those of the nonsteroidal AIs. When interpreting toxicity data, particularly in respect to bone health and cardiovascular risk, it should be borne in mind that for both IES and TEAM, the data accrued are relative to tamoxifen and not to placebo.

Exemestane is relatively well tolerated by the majority of patients; in the IES study, the rate of premature discontinuation of exemestane due to adverse events or patient refusal was $14 \% .{ }^{22}$ However, it should be acknowledged that current evidence suggests a higher rate of noncompliance with hormonal therapy in the routine clinical setting. ${ }^{29,30}$ Patient education and proactive management of toxicity are thus essential components of care.

\section{Vasomotor symptoms}

Hot flashes are a common event in both tamoxifen- and exemestane-treated women. However, the frequency of this adverse event appears to be lower with exemestane. For example, a TEAM substudy, which addressed menopausal symptoms in 1,614 women, observed a peak in hot flashes at 3 months for both tamoxifen and exemestane, with a decline thereafter. ${ }^{31}$ At 12 months, hot flashes were significantly greater in the tamoxifen arm. An increased incidence in vasomotor symptoms has also been reported for the nonsteroidal AIs in comparison to tamoxifen. ${ }^{32,33}$ Furthermore, in patients intolerant of tamoxifen due to hot flashes, switching to exemestane or letrozole was associated with a reduction in severity of almost $50 \%$ at 6 weeks, albeit in a nonblinded study. ${ }^{34}$ On the other hand, the IES did not 
identify a significant difference in vasomotor symptoms..$^{22,35}$ This population, however, is not directly comparable, having already completed 2-3 years of hormonal therapy unlike the upfront comparison addressed in the TEAM substudy. Where vasomotor symptoms are troublesome both lifestyle advice and pharmacological interventions may be of benefit. ${ }^{36}$

\section{Musculoskeletal symptoms}

Musculoskeletal symptoms, primarily classified as arthralgia, have been a consistent finding across trials of AIs. ${ }^{37}$ In the TEAM study, arthralgia was reported in $17.9 \%$ of patients after 2.75 years of upfront exemestane vs $9.2 \%$ in those randomized to tamoxifen $(P<0.001) .{ }^{24}$ In addition, an increased incidence of carpal tunnel syndrome was reported in both the IES ${ }^{22}$ and Arimidex, Tamoxifen, Alone or in Combination (ATAC) ${ }^{38}$ trials. Inflammatory indices, rheumatoid factor, and autoantibodies are typically normal, but characteristic tenosynovial changes have been demonstrated on magnetic resonance imaging. ${ }^{39,40}$ However, the underlying mechanism has yet to be elucidated. Management is typically with simple analgesics and nonsteroidal anti-inflammatory drugs. Nevertheless, in a proportion of patients, musculoskeletal symptoms may be debilitating and necessitate discontinuation of therapy.

\section{Bone density}

There is a clear association between levels of circulating estrogen and the risk of bone density loss and fracture in postmenopausal women. ${ }^{41-43}$ As the third-generation AIs reduce circulating estradiol to virtually undetectable levels, bone loss is a predictable mechanism-related toxicity. In contrast, the use of tamoxifen in postmenopausal women is associated with modest increases in bone density. ${ }^{44}-48$ Therefore, it has been important to address whether any anticancer benefits of exemestane might be diminished by a negative impact on bone health.

A significant increase in the incidence of fractures was observed in the exemestane arm of IES. With a median follow-up of 58 months, $162(7 \%)$ patients in the exemestane group experienced a fracture compared with $115(5 \%)$ patients in the tamoxifen group (odds ratio $=1.45$ [1.13-1.87]; $P=0.003) .{ }^{49} \mathrm{~A}$ subprotocol of IES investigated bone metabolism in more detail in a subset of 206 patients in whom changes in bone density and biochemical markers of bone turnover were measured. ${ }^{49}$ No significant changes in bone density were seen in patients who remained on tamoxifen. However, switching to exemestane was associated with loss of bone density, which was most rapid in the first 6 months with ongoing loss recorded, albeit at a lower rate, in the periods of 6-12 month and 12-24 month. Specifically, in the first 6 months, BMD at the lumbar spine decreased from baseline by $2.7 \%$ (95\% CI: $2.0-3.4 ; P<0.0001)$, with a further decline of $1.0 \%$ ( $95 \%$ CI: $0.39-1.70 ; P=0.002$ ) occurring between 12 and 24 months. Consistent with these reductions in bone density, there was also a significant increase in biochemical markers of bone turnover. A similar pattern was observed in a smaller Italian study, which randomized 70 women who had completed 2-3 years of tamoxifen to either exemestane or continued tamoxifen. Here the switch to exemestane was associated with significant increases in markers of bone turnover and declines in BMD of $2.99 \%(P<0.01)$ at the lumbar spine and $1.92 \%$ $(P<0.01)$ at the femoral neck at 24 months. ${ }^{50}$

In TEAM, with median follow-up of 5.1 years, $5.1 \%$ of patients randomized to upfront exemestane had experienced fractures compared to $3.5 \%$ of those who switched from tamoxifen to exemestane $(P<0.001) .{ }^{25}$ Again, more detailed substudies provide support for a model where exemestane use leads to altered bone metabolism with resultant loss of bone density, ultimately predisposing to fracture. Specifically, a German substudy of the TEAM trial demonstrated significant increases in markers of bone turnover after 3, 6, and 12 months of treatment with exemestane, whereas treatment with tamoxifen resulted in significant reductions in these markers from baseline..$^{51}$ Similarly, a US Oncology TEAM substudy observed significant loss of BMD with exemestane, and this effect was most marked in the first year. ${ }^{52}$

The impact of exemestane on bone health in comparison to placebo rather than tamoxifen was addressed by a Norwegian study, which allocated women with low-risk breast cancer or ductal carcinoma in situ to receive 2 years of exemestane or placebo. In this study, no significant difference was detected in mean annual decline of BMD at the lumbar spine $(2.17 \%$ vs $1.84 \%)$ although there was a significantly greater decline recorded at the femoral neck for exemestane (2.72\% vs $1.48 \% ; P=0.024) .{ }^{53}$ However, the rate of bone loss in the placebo group was higher than expected, perhaps reflecting the high prevalence (88\%) of vitamin D deficiency in this Scandinavian population.

It has been suggested that the androgenic structure of exemestane may promote bone synthesis and thus the overall effect of exemestane on bone may differ from that of the nonsteroidal AIs. ${ }^{54}$ Preclinical data, such as the demonstration of abrogated bone loss in ovariectomized rats treated with exemestane but not letrozole, exist to support this hypothesis. ${ }^{54}$ Furthermore, in a study of 84 healthy postmenopausal women randomized to receive either exemestane, letrozole, or 
anastrozole for 24 weeks, there were no significant differences in markers of bone resorption, but treatment with exemestane was associated with a significant increase from baseline in serum procollagen type I N-terminal propeptide, a marker of bone formation. ${ }^{55}$ However, a separate study of similar design did not replicate this finding with all three AIs having similar effects on biochemical markers of both bone formation and bone resorption. ${ }^{56}$ Taken together with the observed increases in fracture risk attributable to exemestane in both the IES and TEAM trials, a clinically significant difference between exemestane and the nonsteroidal AIs would appear unlikely. ${ }^{57}$

One attraction of the switch/sequential regimens employed by the IES and TEAM trials is that they appear to strike a pragmatic balance between offering improved prevention of cancer recurrence and protecting bone health. However, with appropriate monitoring of BMD, supplementation of calcium and vitamin $\mathrm{D}$, and bisphosphonate therapy where appropriate, this toxicity can be mitigated. ${ }^{58,59}$ Nevertheless, for some women, bone health may be a consideration favoring a sequential or extended adjuvant strategy.

\section{Metabolic events}

The risk of cardiovascular disease increases rapidly in women following the menopause, and this is at least in part attributable to changes in serum lipids. ${ }^{60}$ As in other populations, increasing levels of low-density lipoprotein (LDL) cholesterol are associated with an increased risk of cardiovascular disease in postmenopausal women while higher levels of high-density lipoprotein (HDL) cholesterol exert a protective effect. ${ }^{61,62}$ The effect of exemestane on lipid profiles has been investigated relative to placebo, tamoxifen, and nonsteroidal AIs. Geisler and colleagues randomized 147 postmenopausal women with low-risk breast cancer to receive exemestane $25 \mathrm{mg} / \mathrm{d}$ or placebo for 2 years. ${ }^{53}$ Lipid profiles were determined 6 monthly whilst on treatment and at 6 months and 1 year posttreatment. Exemestane was associated with a small decrease in HDL cholesterol and apolipoprotein A1 levels, but this effect was reversible within 3-12 months of cessation of exemestane.

A Greek substudy of the TEAM trial assessed lipid profiles in 142 patients randomized to upfront tamoxifen or exemestane at baseline and then every 3 months for the first 12 months of treatment and at 18 and 24 months. ${ }^{63}$ There was a fall in total cholesterol levels in both arms, but LDL cholesterol levels fell significantly only in the tamoxifen arm. HDL cholesterol levels decreased across time in both groups, but levels were significantly higher in the tamoxifen arm.
In the overall TEAM study, hyperlipidemia was reported less frequently in patients randomized to the tamoxifencontaining arm $(2.8 \%$ vs $4.7 \%, P<0.001) .{ }^{25}$

In the context of the switching strategy as addressed by IES, exemestane was not associated with a significant increase in hypercholesterolemia vs continued tamoxifen. ${ }^{22}$ However, a small Italian study, which randomized 60 overweight women who had completed 2-3 years of adjuvant tamoxifen to either continued tamoxifen or a switch to exemestane did report a significant increase in LDL cholesterol and fall in HDL cholesterol over the 1-year study period in patients receiving exemestane. ${ }^{64}$ The relative contribution of tamoxifen withdrawal rather than exemestane effect per se is not known but may be particularly relevant for the rise in LDL cholesterol, which was not observed in the comparison of exemestane with placebo described above. ${ }^{53}$ Interestingly, despite these potentially negative changes in lipid profiles, the switch to exemestane was actually associated with beneficial effects on body composition, with a significant decrease in fat mass. Similar changes in lipid profiles were observed in patients who had received 5 years of tamoxifen and were randomized to receive exemestane or observation within the ATENA trial; ${ }^{65}$ there was a significant decrease in HDL cholesterol in the exemestane arm whilst LDL cholesterol levels rose in both arms, likely indicating withdrawal of the beneficial tamoxifen effect.

A comparison of exemestane with letrozole and anastrozole in 90 healthy postmenopausal women reported an increase in atherogenic ratios of LDL cholesterol:HDL cholesterol for exemestane vs the nonsteroidal AIs. ${ }^{56}$

Taken together, these data indicate that exemestane lacks the potentially cardioprotective lipid-altering effects of tamoxifen and, furthermore, may also be associated with modest reductions in levels of HDL cholesterol. However, the clinical significance of these changes is not clear. In particular, a clinically significant elevated risk of cardiovascular events has not been identified in adjuvant trials of exemestane. Similarly, the clinical significance, if any, of the differences reported for exemestane vs the nonsteroidal AIs on lipid profiles is not yet clear.

\section{Cardiovascular/thromboembolic events}

The EBCTCG meta-analysis has demonstrated a nonsignificant trend toward a lower incidence of cardiac deaths in women receiving tamoxifen, ${ }^{5}$ possibly reflecting beneficial effects on lipid profiles. ${ }^{66}$ Conversely, the use of tamoxifen was associated with a nonsignificant increase in the incidence of venous thromboembolic and cerebrovascular events. ${ }^{5}$ 
No significant differences in cardiovascular events (deaths, myocardial infarctions, angina, cerebrovascular accidents), either on treatment or during follow-up, were reported by IES. However, the rate of venous thromboembolic events was significantly higher in patients randomized to continue tamoxifen $(2.3 \%$ vs $1.2 \%, P=0.004) .{ }^{22}$ With median follow-up of 5.1 years, the TEAM study reported a higher incidence of hypertension in the exemestane arm (6\% vs $4.5 \%, P=0.001) .{ }^{25}$ However, there were no differences in the incidence of myocardial ischemia or other serious cardiac events. Again, the risk of venous thromboembolism was significantly higher in the tamoxifen arm $(0.9 \%$ vs $2.0 \%$, $P<0.001)$.

Although individual adjuvant trials of third-generation AIs have not demonstrated statistically significant differences in cardiovascular disease (excluding thromboembolic disease), numerical excesses have been evident in some trials..$^{22,38,67}$ A meta-analysis of adjuvant AI trials has reported an increased rate of grade 3 to 5 cardiovascular events for the use of AIs in comparison to tamoxifen. ${ }^{68}$ However, the absolute risk was low with a number needed to treat for an event to occur of 160-180. It is important that cardiovascular risk factors are managed in the standard manner; it is not appropriate to administer tamoxifen in preference to an AI in order to achieve a putative cardioprotective effect.

\section{Gynecologic toxicity}

Gynecological toxicity is a well-established complication of tamoxifen, in particular, the development of endometrial hyperplasia, polyps, and cysts and a 3-fold increase in the risk of endometrial cancer from 0.6 per 1,000 women years to 1.9 per 1,000 women years. ${ }^{5}$ These toxicities reflect the agonist activity of tamoxifen within the uterus. Although the absolute risk of endometrial cancer remains low, many more women will experience invasive investigations and significant anxiety because of bleeding or discharge whilst on tamoxifen. In contrast, the AIs, with no estrogen agonist activity, have not been associated with an increased risk of endometrial cancer. ${ }^{37}$

The IES trial reported significantly lower rates of vaginal bleeding (5.2\% vs $7.6 \%, P=0.002)$, endometrial hyperplasia $(0.2 \%$ vs $1.2 \%)$, and dilation and curettage $(0.8 \%$ vs $1.8 \%$, $P=0.006)$ in women randomized to exemestane. ${ }^{22}$ The incidence of endometrial cancer was low and did not differ between arms. Transvaginal ultrasound was prospectively planned in a subset of 219 patients enrolled in an IES substudy. ${ }^{69}$ As expected for patients who had received 2-3 years of tamoxifen, there was a high incidence of baseline abnormality with endometrial thickening observed in $62.6 \%$ of patients. The switch to exemestane was associated with a rapid decrease in endometrial thickening over the first 6 months with a slower decline thereafter. No reduction occurred in patients who continued to receive tamoxifen. However, following cessation of tamoxifen, a decline occurred similar to that seen in those patients switched to exemestane. Patients participating in the IES Quality of Life substudy reported less vaginal discharge if randomized to exemestane. ${ }^{35}$ Loss of libido was common in both arms and did not differ significantly.

Within the TEAM study, those patients randomized to tamoxifen experienced significantly higher rates of vaginal bleeding (3.1\% vs $1.6 \%)$ and endometrial hyperplasia ( $2 \%$ vs $0 \%$ ). In addition, there was a numerical, but not statistically significant, excess of endometrial cancer in the tamoxifen arm. ${ }^{24}$ A US Oncology substudy of 1,614 women treated within TEAM has provided a detailed assessment of menopausal symptoms occurring during the first year of treatment; exemestane use was associated with less vaginal discharge but with more vaginal dryness and a higher frequency of reduced libido. ${ }^{31}$

\section{Predictive biomarkers for exemestane}

Initial hypothesis-generating data suggested that ER-positive/ PR-negative cancers may exhibit greater sensitivity to aromatase inhibition than to tamoxifen. ${ }^{70}$ However, this has not been supported by subsequent data. For example, exemestane appeared to be equally effective in the PR-positive and PR-negative subsets in an unplanned analysis of the IES data set. ${ }^{21}$ Furthermore, in a prospectively planned analysis of the TEAM data set, PR was prognostic but did not predict preferential benefit for tamoxifen over exemestane. ${ }^{71}$ Similar prognostic but not predictive significance for PR expression has been identified in the BIG $1-98^{72}$ and Trans-ATAC ${ }^{73}$ studies.

Bartlett and colleagues have presented data based on a subset of 4,308 patients in the TEAM trial for whom biomarker data could be obtained. ${ }^{74}$ Specifically, in a prospectively planned analysis, they investigated ER, PR, human epidermal growth factor receptor (HER) 1-3, and Ki67 as continuous variables relative to DFS for the first 2.75 years of the TEAM trial (ie, the upfront tamoxifen vs exemestane comparison). In this analysis, patients who were HER $2 / 3$ positive derived no additional benefit from exemestane over tamoxifen [HR 1.13 (95\% CI: $0.82-1.55$ ). In contrast, the additional benefit of exemestane appeared to be confined to 
those with HER2/3-negative tumors ( $\mathrm{HR}=0.69$ [95\% CI: 0.53-0.88]). This is consistent with HER2/3 tumors having an intrinsic resistance to hormonal therapy. A similar pattern was identified in the Trans-ATAC dataset albeit with a low number of events occurring. ${ }^{73}$ How this may be modified by the widespread use of adjuvant HER2-directed therapies is not known.

Thus specific predictive markers to select patients who may preferentially benefit from early AI are lacking. However, in addition to considering putative predictive markers, current prognostic markers such as nodal status, grade, size, age, and PR levels might help to select those patients at higher risk of early recurrence, who will achieve a greater absolute benefit with upfront AI therapy. For example, Bartlett has presented data based on the Her2/3-negative subset in the TEAM study, showing a number needed to treat to prevent an early relapse of 160 for those patients defined as lower risk vs 20 for those in a higher risk category. ${ }^{74}$

\section{Adjuvant exemestane in premenopausal women}

As explained above, AI use is contraindicated in women with functioning ovaries. Nevertheless, given the benefits in DFS and/or OS reported for certain AI strategies in postmenopausal women, it has been important to ascertain whether similar benefits might accrue in premenopausal women who are rendered menopausal by ovarian oblation or suppression. This was addressed in the Austrian Breast and Colorectal Cancer Study Group-12 trial in which 1,803 premenopausal women were randomized to receive ovarian suppression with goserelin with either tamoxifen or anastrozole. ${ }^{75}$ With median follow-up of 47.8 months, there was no significant difference in DFS between the anastrozole and the tamoxifen groups (HR for disease progression in the anastrozole group, 1.10; 95\% CI: $0.78-1.53 ; P=0.59)$. Two ongoing trials will address this question further. The Suppression of Ovarian Function trial randomizes women to tamoxifen alone or to goserelin with either tamoxifen or exemestane. The Tamoxifen and Exemestane trial randomizes women to goserelin with either tamoxifen or exemestane. At present, the adjuvant use of ovarian suppression and an AI must be considered an investigational approach.

A separate issue is adjuvant AI use in premenopausal women who have experienced chemotherapy-induced amenorrhea. The majority of women who receive adjuvant chemotherapy will become amenorrheic during treatment with the incidence of permanent chemotherapy-induced amenorrhea increasing with age such that permanent cessation of menses is observed in most women over the age of $40 .{ }^{76}$ However, it is clear that chemotherapy-induced amenorrhea does not equate to permanent cessation of ovarian function and that aromatase inhibition may contribute to recovery of ovarian function via hypothalamic-pituitary feedback. For example, 12 of 45 women (median age 47, range 39-52) treated at the Royal Marsden who had been amenorrheic for 6 months postchemotherapy and who had biochemical evidence of ovarian suppression subsequently recovered ovarian function after commencing on $\mathrm{AI} .{ }^{77}$ This included one patient who became pregnant without recovery of menstruation and another patient with biochemical recovery of ovarian function without menstruation. Thus, in this case, series AI use would appear to be potentially harmful in a quarter of such patients. For this reason, AIs should be used with great caution in this population as addressed by recent guidelines. ${ }^{77,78}$

\section{Conclusion}

The weight of evidence indicates that strategies that incorporate third-generation AIs are associated with improved DFS; however, the optimal strategy for incorporation of AIs into adjuvant therapy remains a matter of controversy. For those patients at the lowest risk of relapse, the absolute benefit may be minimal and tamoxifen monotherapy considered acceptable. On the other hand, for those patients at high risk of relapse, the use of an AI should be considered. The attempts to define subgroups that may gain most from an upfront AI have used complex modeling of biological and clinical risk features, which have resulted in defining patients who have a larger absolute benefit from the AI, rather than a relative increased benefit compared to tamoxifen, due to a higher clinical risk and with biological features suggesting generally less hormone-sensitive tumors. This is in contrast to the MA17 and IES trials which appear to show an increased benefit, and for some patients gains in OS, from an AI in populations that are enriched for hormone sensitivity by enrolling patients still disease free after 5 years or 2.75 years of tamoxifen, respectively.

Rather than considerations of efficacy, treatment choices in an individual patient may be influenced by the different toxicity profiles with more gynecological toxicity and venous thromboembolic disease occurring with tamoxifen vs arthralgia and the need for monitoring and treatment of bone health with aromatase inhibition. In this context, it may well be that a sequential strategy that should minimize toxicities would be the optimum choice.

As regards the comparative efficacy and toxicity of exemestane vs nonsteroidal AIs, these agents do have distinct 
pharmacokinetic and pharmacodynamic profiles, but the clinical significance of these differences will be best addressed by the ongoing comparative trials such as MA27 rather than by cross-trial comparisons. At present, exemestane should be used according to trial data as reflected in the product label.

\section{Disclosure}

The authors report no conflicts of interest in this work.

\section{References}

1. Cancer Research UK. CancerStats: Cancer Worldwide. Available from: http://info.cancerresearchuk.org/cancerstats/world/commoncancers/ index.htm. Accessed 2010 Jan 10.

2. Beatson GT. On treatment of inoperable cases of carcinoma of the mamma: suggestions for a new method of treatment with illustrative cases. Lancet. 1896;2:162-165.

3. Santen RJ, Brodie H, Simpson ER, Siiteri PK, Brodie A. History of aromatase: saga of an important biological mediator and therapeutic target. Endocr Rev. 2009;30(4):343-375.

4. Jordan VC. Tamoxifen: a most unlikely pioneering medicine. Nat Rev Drug Discov. 2003;2(3):205-213.

5. Early Breast Cancer Trialists' Collaborative Group (EBCTCG). Effects of chemotherapy and hormonal therapy for early breast cancer on recurrence and 15-year survival: an overview of the randomized trials. Lancet. 2005;365(9472):1687-1717.

6. Miller WR. Aromatase inhibitors: mechanism of action and role in the treatment of breast cancer. Semin Oncol. 2003;30(4 Suppl 14):3-11.

7. Geisler J, King N, Anker G, et al. In vivo inhibition of aromatization by exemestane, a novel irreversible aromatase inhibitor, in postmenopausal breast cancer patients. Clin Cancer Res. 1998;4(9):2089-2093.

8. Geisler J, Haynes B, Anker G, Dowsett M, Lonning PE. Influence of letrozole and anastrozole on total body aromatization and plasma estrogen levels in postmenopausal breast cancer patients evaluated in a randomized, cross-over study. J Clin Oncol. 2002;20(3):751-757.

9. Nabholtz JM, Bonneterre J, Buzdar A, Robertson JF, Thurlimann B. Anastrozole (Arimidex) versus tamoxifen as first-line therapy for advanced breast cancer in postmenopausal women: survival analysis and updated safety results. Eur J Cancer. 2003;39(12):1684-1689.

10. Mouridsen H, Gershanovich M, Sun Y, et al. Phase III study of letrozole versus tamoxifen as first-line therapy of advanced breast cancer in postmenopausal women: analysis of survival and update of efficacy from the International Letrozole Breast Cancer Group. J Clin Oncol. 2003; 21(11):2101-2109.

11. Paridaens RJ, Dirix LY, Beex LV, et al. Phase III study comparing exemestane with tamoxifen as first-line hormonal treatment of metastatic breast cancer in postmenopausal women: the European Organisation for Research and Treatment of Cancer Breast Cancer Cooperative Group. J Clin Oncol. 2008;26(30):4883-4890.

12. Evans TR, Di Salle E, Ornati G, et al. Phase I and endocrine study of exemestane (FCE 24304), a new aromatase inhibitor, in postmenopausal women. Cancer Res. 1992;52(21):5933-5939.

13. Johannessen DC, Engan T, Di Salle E, et al. Endocrine and clinical effects of exemestane (PNU 155971), a novel steroidal aromatase inhibitor, in postmenopausal breast cancer patients: a phase I study. Clin Cancer Res. 1997;3(7):1101-1108.

14. Lonning P, Pfister C, Martoni A, Zamagni C. Pharmacokinetics of thirdgeneration aromatase inhibitors. Semin Oncol. 2003;30(4 Suppl 14): 23-32.

15. Pharmacia Ltd. Exemestane - Summary of product characteristics. Available from: http://emc.medicines.org.uk/medicine/2484/SPC/ Aromasin/. Accessed 2010 Mar 16.
16. Jones S, Vogel C, Arkhipov A, et al. Multicenter, phase II trial of exemestane as third-line hormonal therapy of postmenopausal women with metastatic breast cancer. Aromasin Study Group. J Clin Oncol. 1999;17(11):3418-3425.

17. Lonning PE, Bajetta E, Murray R, et al. Activity of exemestane in metastatic breast cancer after failure of nonsteroidal aromatase inhibitors: a phase II trial. J Clin Oncol. 2000;18(11):2234-2244.

18. Kvinnsland S, Anker G, Dirix LY, et al. High activity and tolerability demonstrated for exemestane in postmenopausal women with metastatic breast cancer who had previously failed on tamoxifen treatment. Eur $J$ Cancer. 2000;36(8):976-982.

19. Kaufmann M, Bajetta E, Dirix LY, et al. Exemestane is superior to megestrol acetate after tamoxifen failure in postmenopausal women with advanced breast cancer: results of a phase III randomized doubleblind trial. The Exemestane Study Group. J Clin Oncol. 2000;18(7): 1399-1411.

20. Paridaens R, Dirix L, Lohrisch C, et al. Mature results of a randomized phase II multicenter study of exemestane versus tamoxifen as first-line hormone therapy for postmenopausal women with metastatic breast cancer. Ann Oncol. 2003;14(9):1391-1398.

21. Coombes RC, Hall E, Gibson LJ, et al. A randomized trial of exemestane after two to three years of tamoxifen therapy in postmenopausal women with primary breast cancer. $N$ Engl J Med. 2004;350(11):1081-1092.

22. Coombes RC, Kilburn LS, Snowdon CF, et al. Survival and safety of exemestane versus tamoxifen after 2-3 years' tamoxifen treatment (Intergroup Exemestane Study): a randomized controlled trial. Lancet. 2007;369(9561):559-570.

23. Coombes R, Kilburn L, Beare S, Snowdon C, Bliss J. Survival and safety post study treatment completion: an updated analysis of the Intergroup Exemestane Study (IES) - submitted on behalf of the IES Investigators. Eur J Cancer Supps. 2009;7(2):263.

24. Jones SE, Seynaeve C, Hasenburg A, et al. Results of the first planned analysis of the TEAM (tamoxifen exemestane adjuvant multinational) prospective randomized phase III trial in hormone sensitive postmenopausal early breast cancer. Cancer Res. 2009;69 Suppl 2. Abstract 15 .

25. Rea D, Hasenburg A, Seynaeve C, et al. Five years of exemestane as initial therapy compared to 5 years of tamoxifen followed by exemestane: the TEAM Trial, a prospective, randomized, phase III trial in postmenopausal women with hormone-sensitive early breast cancer. Cancer Res. 2009;69 Suppl 24. Abstract 11.

26. Mouridsen H, Giobbie-Hurder A, Goldhirsch A, et al. Letrozole therapy alone or in sequence with tamoxifen in women with breast cancer. N Engl J Med. 2009;361(8):766-776.

27. Mamounas EP, Jeong JH, Wickerham DL, et al. Benefit from exemestane as extended adjuvant therapy after 5 years of adjuvant tamoxifen: intention-to-treat analysis of the National Surgical Adjuvant Breast and Bowel Project B-33 trial. J Clin Oncol. 2008;26(12):1965-1971.

28. Phase III randomized adjuvant study of exemestane versus anastrozole in postmenopausal women with receptor-positive primary breast cancer (CAN-NCIC-MA27). Available from: http://www.cancer.gov/clinicaltrials/ ft-CAN-NCIC-MA27. Accessed 2010 Mar 16.

29. Chlebowski RT, Geller ML. Adherence to endocrine therapy for breast cancer. Oncology. 2006;71(1-2):1-9.

30. Partridge AH, LaFountain A, Mayer E, Taylor BS, Winer E, Asnis-Alibozek A. Adherence to initial adjuvant anastrozole therapy among women with early-stage breast cancer. J Clin Oncol. 2008; 26(4):556-562.

31. Jones SE, Cantrell J, Vukelja S, et al. Comparison of menopausal symptoms during the first year of adjuvant therapy with either exemestane or tamoxifen in early breast cancer: report of a Tamoxifen Exemestane Adjuvant Multicenter trial substudy. J Clin Oncol. 2007; 25(30):4765-4771.

32. Fallowfield L, Cella D, Cuzick J, Francis S, Locker G, Howell A. Quality of life of postmenopausal women in the Arimidex, Tamoxifen, Alone or in Combination (ATAC) Adjuvant Breast Cancer Trial. J Clin Oncol. 2004;22(21):4261-4271. 
33. Thurlimann B, Keshaviah A, Coates AS, et al. A comparison of letrozole and tamoxifen in postmenopausal women with early breast cancer. N Engl J Med. 2005;353(26):2747-2757.

34. Thomas R, Williams M, Marshall C, Walker L. Switching to letrozole or exemestane improves hot flushes, mood and quality of life in tamoxifen intolerant women. Br J Cancer. 2008;98(9):1494-1499.

35. Fallowfield LJ, Bliss JM, Porter LS, et al. Quality of life in the intergroup exemestane study: a randomized trial of exemestane versus continued tamoxifen after 2 to 3 years of tamoxifen in postmenopausal women with primary breast cancer. J Clin Oncol. 2006;24(6): 910-917.

36. Hickey M, Saunders C, Partridge A, Santoro N, Joffe H, Stearns V. Practical clinical guidelines for assessing and managing menopausal symptoms after breast cancer. Ann Oncol. 2008;19(10):1669-1680.

37. Mouridsen HT. Incidence and management of side effects associated with aromatase inhibitors in the adjuvant treatment of breast cancer in postmenopausal women. Curr Med Res Opin. 2006;22(8): 1609-1621.

38. Buzdar A, Howell A, Cuzick J, et al. Comprehensive side-effect profile of anastrozole and tamoxifen as adjuvant treatment for early-stage breast cancer: long-term safety analysis of the ATAC trial. Lancet Oncol. 2006;7(8):633-643.

39. Morales L, Pans S, Paridaens R, et al. Debilitating musculoskeletal pain and stiffness with letrozole and exemestane: associated tenosynovial changes on magnetic resonance imaging. Breast Cancer Res Treat. 2007;104(1):87-91.

40. Henry NL, Giles JT, Stearns V. Aromatase inhibitor-associated musculoskeletal symptoms: etiology and strategies for management Oncology (Williston Park). 2008;22(12):1401-1408.

41. Rogers A, Saleh G, Hannon RA, Greenfield D, Eastell R. Circulating estradiol and osteoprotegerin as determinants of bone turnover and bone density in postmenopausal women. J Clin Endocrinol Metab. 2002; 87(10): 4470-4475.

42. Cummings SR, Browner WS, Bauer D, et al. Endogenous hormones and the risk of hip and vertebral fractures among older women. Study of Osteoporotic Fractures Research Group. NEngl J Med. 1998;339(11): 733-738.

43. Chapurlat RD, Garnero P, Breart G, Meunier PJ, Delmas PD. Serum estradiol and sex hormone-binding globulin and the risk of hip fracture in elderly women: the EPIDOS study. J Bone Miner Res. 2000;15(9): 1835-1841.

44. Powles TJ, Hickish T, Kanis JA, Tidy A, Ashley S. Effect of tamoxifen on bone mineral density measured by dual-energy $\mathrm{x}$-ray absorptiometry in healthy premenopausal and postmenopausal women. J Clin Oncol. 1996;14(1):78-84.

45. Love RR, Mazess RB, Barden HS, et al. Effects of tamoxifen on bone mineral density in postmenopausal women with breast cancer. $N$ Engl J Med. 1992;326(13):852-856.

46. Resch A, Biber E, Seifert M, Resch H. Evidence that tamoxifen preserves bone density in late postmenopausal women with breast cancer. Acta Oncol. 1998;37(7-8):661-664.

47. Kristensen B, Ejlertsen B, Dalgaard P, et al. Tamoxifen and bone metabolism in postmenopausal low-risk breast cancer patients: a randomized study. J Clin Oncol. 1994;12(5):992-997.

48. Grey AB, Stapleton JP, Evans MC, Tatnell MA, Ames RW, Reid IR. The effect of the antiestrogen tamoxifen on bone mineral density in normal late postmenopausal women. Am J Med. 1995;99(6): 636-641.

49. Coleman RE, Banks LM, Girgis SI, et al. Skeletal effects of exemestane on bone-mineral density, bone biomarkers, and fracture incidence in postmenopausal women with early breast cancer participating in the Intergroup Exemestane Study (IES): a randomized controlled study. Lancet Oncol. 2007;8(2):119-127.

50. Gonnelli S, Cadirni A, Caffarelli C, et al. Changes in bone turnover and in bone mass in women with breast cancer switched from tamoxifen to exemestane. Bone. 2007;40(1):205-210.
51. Hadji P, Ziller M, Kieback DG, et al. Effects of exemestane and tamoxifen on bone health within the Tamoxifen Exemestane Adjuvant Multicentre (TEAM) trial: results of a German, 12-month, prospective, randomized substudy. Ann Oncol. 2009;20(7):1203-1209.

52. Jones S, Stokoe C, Sborov M, et al. The effect of tamoxifen or exemestane on bone mineral density during the first 2 years of adjuvant treatment of postmenopausal women with early breast cancer. Clin Breast Cancer. 2008;8(6):527-532.

53. Geisler J, Lonning PE, Krag LE, et al. Changes in bone and lipid metabolism in postmenopausal women with early breast cancer after terminating 2-year treatment with exemestane: a randomized, placebocontrolled study. Eur J Cancer. 2006;42(17):2968-2975.

54. Goss PE, Qi S, Cheung AM, Hu H, Mendes M, Pritzker KP. Effects of the steroidal aromatase inhibitor exemestane and the nonsteroidal aromatase inhibitor letrozole on bone and lipid metabolism in ovariectomized rats. Clin Cancer Res. 2004;10(17):5717-5723.

55. Goss PE, Hadji P, Subar M, Abreu P, Thomsen T, Banke-Bochita J. Effects of steroidal and nonsteroidal aromatase inhibitors on markers of bone turnover in healthy postmenopausal women. Breast Cancer Res. 2007;9(4):R52.

56. McCloskey EV, Hannon RA, Lakner G, et al. Effects of third generation aromatase inhibitors on bone health and other safety parameters: results of an open, randomized, multi-centre study of letrozole, exemestane and anastrozole in healthy postmenopausal women. Eur J Cancer. 2007; 43(17):2523-2531.

57. McCloskey E. Effects of third-generation aromatase inhibitors on bone. Eur J Cancer. 2006;42(8):1044-1051.

58. Hillner BE, Ingle JN, Chlebowski RT, et al. American Society of Clinical Oncology 2003 update on the role of bisphosphonates and bone health issues in women with breast cancer. J Clin Oncol. 2003;21(21): 4042-4057.

59. Reid DM, Doughty J, Eastell R, et al. Guidance for the management of breast cancer treatment-induced bone loss: a consensus position statement from a UK Expert Group. Cancer Treat Rev. 2008;34 Suppl 1: S3-S18.

60. Ewer MS, Gluck S. A woman's heart: the impact of adjuvant endocrine therapy on cardiovascular health. Cancer. 2009;115(9):1813-1826.

61. Kannel WB. Metabolic risk factors for coronary heart disease in women: perspective from the Framingham Study. Am Heart J. 1987;114(2): 413-419.

62. Bass KM, Newschaffer CJ, Klag MJ, Bush TL. Plasma lipoprotein levels as predictors of cardiovascular death in women. Arch Intern Med. 1993;153(19):2209-2216.

63. Markopoulos C, Polychronis A, Dafni U, et al. Lipid changes in breast cancer patients on exemestane treatment: final results of the TEAM Greek substudy. Ann Oncol. 2009;20(1):49-55.

64. Francini G, Petrioli R, Montagnani A, et al. Exemestane after tamoxifen as adjuvant hormonal therapy in postmenopausal women with breast cancer: effects on body composition and lipids. Br J Cancer. 2006;95(2): $153-158$.

65. Markopoulos C, Dafni U, Misitzis J, et al. Extended adjuvant hormonal therapy with exemestane has no detrimental effect on the lipid profile of postmenopausal breast cancer patients: final results of the ATENA lipid substudy. Breast Cancer Res. 2009;11(3):R35

66. Grey AB, Stapleton JP, Evans MC, Reid IR. The effect of the antiestrogen tamoxifen on cardiovascular risk factors in normal postmenopausal women. J Clin Endocrinol Metab. 1995;80(11): 3191-3195.

67. Mouridsen H, Keshaviah A, Coates AS, et al. Cardiovascular adverse events during adjuvant endocrine therapy for early breast cancer using letrozole or tamoxifen: safety analysis of BIG 1-98 trial. J Clin Oncol. 2007;25(36):5715-5722.

68. Cuppone F, Bria E, Verma S, et al. Do adjuvant aromatase inhibitors increase the cardiovascular risk in postmenopausal women with early breast cancer? Meta-analysis of randomized trials. Cancer. 2008;112(2): $260-267$. 
69. Bertelli G, Hall E, Ireland E, et al. Long-term endometrial effects in postmenopausal women with early breast cancer participating in the Intergroup Exemestane Study (IES) - a randomized controlled trial of exemestane versus continued tamoxifen after 2-3 years tamoxifen. Ann Oncol. 2010;21(3):498-505.

70. Dowsett M, Cuzick J, Wale C, Howell T, Houghton J, Baum M. Retrospective analysis of time to recurrence in the ATAC trial according to hormone receptor status: an hypothesis-generating study. $J$ Clin Oncol. 2005;23(30):7512-7517.

71. Bartlett J, Brookes C, Billingham L, et al. A prospectively planned pathology study within the TEAM trial confirms that progesterone receptor expression is prognostic but is not predictive for differential response to exemestane versus tamoxifen. Cancer Res. 2009;69 Suppl 2. Abstract 81.

72. Viale G, Regan MM, Maiorano E, et al. Prognostic and predictive value of centrally reviewed expression of estrogen and progesterone receptors in a randomized trial comparing letrozole and tamoxifen adjuvant therapy for postmenopausal early breast cancer: BIG 1-98. J Clin Oncol. 2007;25(25):3846-3852.

73. Dowsett M, Allred C, Knox J, et al. Relationship between quantitative estrogen and progesterone receptor expression and human epidermal growth factor receptor 2 (HER-2) status with recurrence in the Arimidex, Tamoxifen, Alone or in Combination trial. J Clin Oncol. 2008;26(7): 1059-1065.
74. Bartlett J, Brookes C, Robson T, et al. The TEAM trial pathology study identifies potential prognostic and predictive biomarker models for postmenopausal patients treated with endocrine therapy. Cancer Res. 2009;69 Supp1 24. Abstract 75.

75. Gnant M, Mlineritsch B, Schippinger W, et al. Endocrine therapy plus zoledronic acid in premenopausal breast cancer. N Engl J Med. 2009; 360(7):679-691.

76. Walshe JM, Denduluri N, Swain SM. Amenorrhea in premenopausal women after adjuvant chemotherapy for breast cancer. J Clin Oncol. 2006;24(36):5769-5779.

77. Smith IE, Dowsett M, Yap YS, et al. Adjuvant aromatase inhibitors for early breast cancer after chemotherapy-induced amenorrhea: caution and suggested guidelines. J Clin Oncol. 2006;24(16):2444-2447.

78. NCCN Clinical Practice Guidelines in Oncology: Breast Cancer. Available from: http://www.nccn.org/professionals/physician_gls/PDF/ breast.pdf. Accessed 2010 Mar 23.

\section{Publish your work in this journal}

Breast Cancer: Targets and Therapy is an international, peerreviewed open access journal focusing on breast cancer research, identification of therapeutic targets and the optimal use of preventative and integrated treatment interventions to achieve improved outcomes, enhanced survival and quality of life for the cancer patient.
View the full aims and scopes of this journal here. The manuscript management system is completely online and includes a very quick and fair peer-review system, which is all easy to use. Visit http:// www.dovepress.com/testimonials.php to read real quotes from published authors.

Submit your manuscript here: http://www.dovepress.com/breast-cancer---targets-and-therapy-journal 